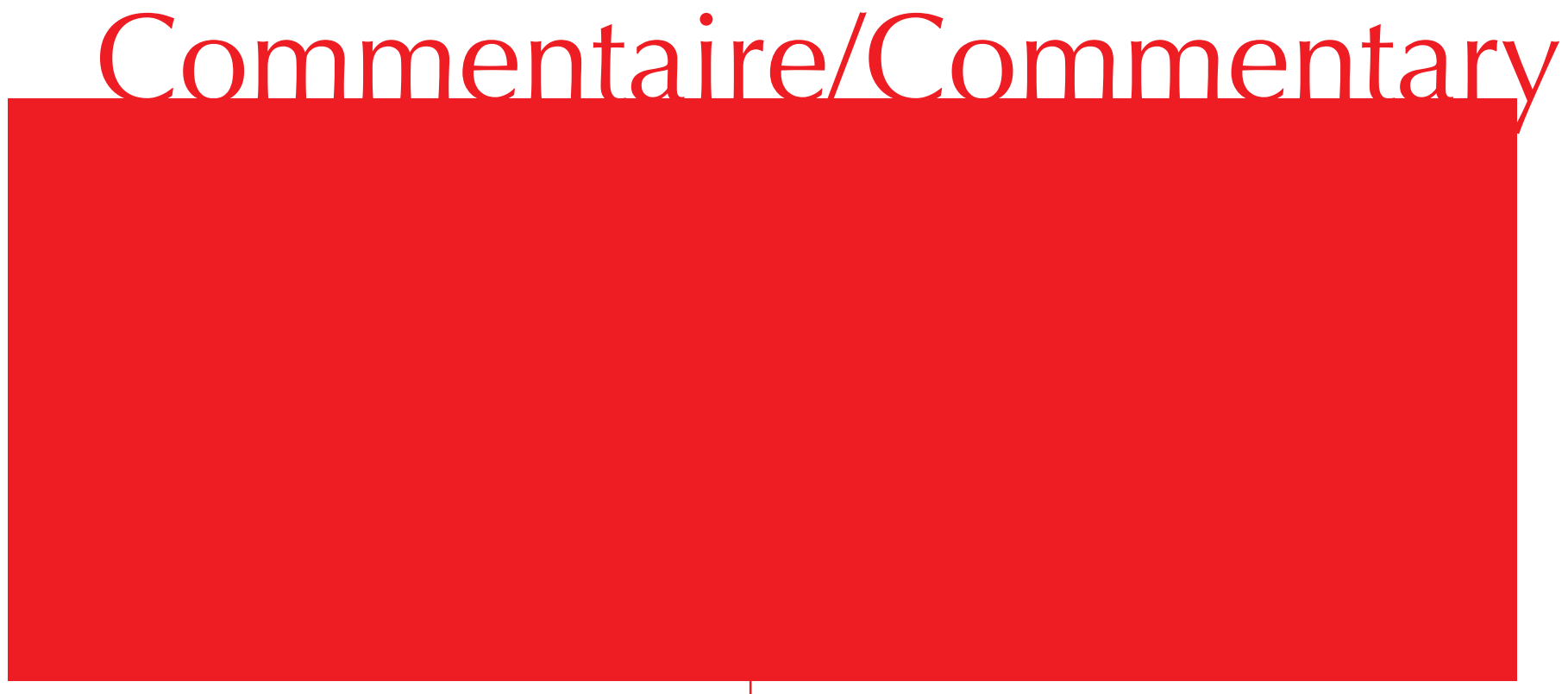

\section{Pourquoi un Laboratoire de pratiques sociales et d'éducation populaire en santé à l'École des sciences infirmières?}

\section{HÉLÈNE LAPERRIÈRE}

\section{Introduction}

Inspirée de l'expérience brésilienne,[1-4] la vision d'un laboratoire de pratiques sociales et d'éducation populaire en sciences infirmières a émergé en 2008 comme un format de travail inclusif des expériences de pratiques sociosanitaires locales dans les recherches terrain.[5] Pourquoi un laboratoire? II y a une conscience de plus en plus grande que la santé s'insère dans des contextes locaux traversés par des dimensions sociopolitiques et socioculturelles. La production des connaissances sur les pratiques s'ancre dans les contextes où elles ont lieu. La multiplicité des acteurs exige que les milieux académiques, de pratiques et d'actions locales puissent échanger pour produire des connaissances qui leur soient reconnaissables (ex. organismes communautaires, coalitions, coopératives, associations de la société civile populaires).[6,7] Les savoirs doivent être pertinents à leurs ancrages sociopolitiques[8] et à leur forme de production des connaissances « hybrides » (savoirs populaires, professionnels et académiques).

Le laboratoire rappelle la notion de Donald Campbell[9] sur une société qui expérimente [an « experimenting society »]. La démarche méthodologique suit la "prospective». Une sociologue française a rappelé le terme lors du Colloque Cérisy 2010 : Prendre soin : savoirs, pratiques, nouvelles perspectives (France, 11 juin-21 juin 2010). Elle vise à regarder le futur en bricolant des scénarios significatifs pour les acteurs locaux concernés à partir des éléments d'analyse disponibles dans le présent : un regard sur les tendances et les mouvements émergents, plutôt que la généralisation à partir d'un cadre théorique prescriptif « divinatoire ». La prospective est la science de l'action. [10] 
Le « Laboratoire » implique des séminaires, des pratiques exploratoires, des actions concertées, des forums d'échange et des publications de différent format pour une démocratisation des savoirs. La démocratisation se concrétise par l'engagement communautaire de proximité dans des contextes sociopolitiques et socioculturels incertains.[11] Les acteurs développent des compétences pratiques aptes à intégrer les expériences de leur propre trajectoire personnelle[12] et identitaire[13] dans la construction des méthodologies de recherche. Les produits peuvent être l'étude avec des milieux communautaires, les pratiques d'intervention et de formation, les actions de la société civile en lien avec le socio-sanitaire local.

Les méthodologies se composent d'approches contextualisées aux milieux et situations émergentes. La boîte à outils de méthodes contient les approches de proximité d'éducation populaire, les moyens audio-visuels, l'ethnographie collaborative, la recherche-action intégrale, la recherche communautaire et la systématisation des expériences réflexives et auto-ethnographiques. Les expérimentations terrain soulignent des caractéristiques souvent négligées: (a) la participation démocratique dans les prises de décision du processus; (b) l'imprévisibilité dans les programmes sociosanitaires; (c) la multiplicité des acteurs et la verticalité

Tableau 1: Le contraste des modes de connaissances scientifiques

\section{LA TRANSFORMATION DE LA SOCIÉTÉ : LA MODERNITÉ DANS UN MODE 1}

La connaissance scientifique triomphante.

LA SOCIÉTÉ CONNAISSANTE : La croyance dans des formes de prévision sociale engendrées par un déterminisme technologique.

De l'application des connaissances...

LA SCIENCE MODE 1

Culture de science : autonomiste, réductrice à ses intérêts, autoréférentielle.

\section{LA TRANSFORMATION DE LA SOCIÉTÉ : AU-DELÀ DE LA MODERNITÉ, DANS UN MODE 2}

Des systèmes plus ouverts de production des connaissances qui tiennent compte du développement de la complexité et de l'incertitude sociétale et scientifique.

LA SOCIÉTÉ DE RISQUE : Développement de la volatilité intellectuelle et sociale... Une coévolution de la société et de la science.

à l'implication dans la production

LA SCIENCE MODE 2

$>$... une culture de recherche, plus populiste, pluraliste et ouverte.

Le contexte parle :

Les dangers des « contextualisations faibles » : au-delà des programmes de recherche dirigée.

Comment chercher la qualité de la recherche dans les situa-

tions complexes créées par le Mode-2 ?

Connaissance de la fiabilité par la mise en contexte :

Les universités et les centres de recherche défendent la possession d'une connaissance objective et désintéressée parce qu'ils se réclament des producteurs de connaissance libre des contextes...

Le présupposé et la défense des enclaves restreints de production officielle des connaissances.
... tandis que le Mode 2 est transgressif des traditions par sa réflexivité autocritique, son éclectisme et sa mise radicale dans les contextes de production.

L'acceptation du fait de la prolifération « d'industries des connaissances ».

La connaissance générée dans «le contexte d'application».

L'ENJEU ÉPISTÉMOLOGIQUE

La science se déplace à l'agora,

L'expertise est socialement distribuée:

La montée de l'individu comme preneur de décisions rationnelles.

Une science revisitée :

UNE RECONSTRUCTION DE L'AUTORITÉ SCIENTIFIQUE

[Tableau créé en collaboration avec Ricardo Zúñiga] 
(relations de contrôle de pouvoir); (d) la dangerosité oubliée de l'insertion des professionnels dans la communauté (politiques locales, criminalité, contraintes cachées); (e) le potentiel de la communauté évaluative émergente (société civile); et (f) l'engagement infirmier dans la communauté et la société civile (médiation entre théories du programme et conditions locales d'implantation). La démocratisation des sites de production des connaissances nous pousse donc vers un virage.

\section{Production des connaissances : Mode 2}

La participation communautaire à la recherche appelle à un nouveau rapport dans la production et la mise en œuvre des connaissances. Deux modèles de production de connaissances scientifiques représentent deux conceptions du rapport de la science comme produit intellectuel collectif aux conditions sociales de cette production. Ils correspondent à un nouveau rapport entre de récentes conceptions de société et de recherche[8,14] pouvant être pertinentes pour la prise de décision en santé.[15] Nowotny, Scott et Gibbons[8] évoquent l'émergence d'un "Mode 2 », dans lequel l'expansion de la science par la diversification des sites et des réseaux de production de connaissances s'incarne dans de nouvelles formes institutionnelles où les scientifiques et les non-scientifiques participent à la création jusqu'au réaménagement des connaissances.

Dépassant les ajustements marginaux de la logique scientifique traditionnelle des organisations universitaires de recherche du "Mode 1 », ce processus social de contextualisation du savoir accroît l'utilisation rapide des connaissances parmi les acteurs sociaux visant une finalité pragmatique plutôt que la simple valeur de production en soi. Ricardo Zúniga et moi avons construit le Tableau I pour récapituler les différenciations essentielles entre les deux modes de production des connaissances présentés par Nowotny et al.[8] et Gibbons, Limoges, Nowotny et al.[14]

Mais, la diffusion et le transfert des connaissances laissent les mécanismes d'imposition en arrière-plan de l'analyse. II semble plus adéquat de parler de "colonisation interne » que de «socialisation ».

\section{Encourager une conscience préventive des colonisations}

L'usage de la notion de " colonisation » suggère un raccourci terminologique, qui fusionne la transmission véhiculée dans la socialisation et le décalage de pouvoir implicite dans cette diffusion. Elle souligne ainsi le changement social prétendument autonome, qui demeure cependant le produit d'une relation inégalitaire de "promotion »proche de l'incitation, la conscientisation, la formation... bref, l'inculcation. L'anthropologue brésilien Lovisolo[16] a ironisé cette approche: nous ne pouvons accepter l'action autonome des communautés, nous devons les changer et les transformer à notre image.

Il y a deux types de colonisation. La première est la colonisation internationale, avec un discours sous-jacent de "conscientisation », pour contrer le présupposé tacite que le sous-développement économique d'un pays n'est que le reflet d'un sous-développement social et intellectuel [ "tiers-monde», « en voie de développement» ou encore " émergent »]. Les pays donateurs créent des produits théoriques qu'ils exporteront avec l'affirmation que l'universalité scientifique garantit leur pertinence. L'autre est la colonisation interne, analysée dans les années 60 par les mexicains Pablo Gonzalez Casanova[17] et Rodolfo Stavenhagen[18], dans laquelle l'unité d'une nation se fonde sur l'implantation de l’homogénéité, même avec des cultures distinctes et diverses au niveau régional, culturel, ethnique et socioéconomique. Elle est le complément pratique de la première, en transférant l'action répressive aux appareils d'État locaux. Les inégalités sociopolitiques intranationales sont inséparables de ce mouvement d'incitation, métaphoriquement soutenu par l'image de l'européen développé en opposition aux populations primitives et archaïques.

La thèse sur la sociologie de l'exploitation [17] offre des pistes de lecture critique. La notion de développement dans la colonisation interne est intimement reliée à un mouvement pacifique, mais continu, en direction d'un changement pour "quelque chose de meilleur ». Souvent présenté comme une valorisation de l'autonomie et de la capacité d'agir, le processus de changement n'est que le produit d'une direction souhaitée par les esprits "lucides ». Ils voient leur propre modèle national comme étant le modèle idéal et universel - demeurant sans définition autre qu'idéologique. Ils s'appuient sur la conviction profonde que tout changement, qui rapprocherait la situation locale du modèle importé, est validé comme progrès.[17] Les inégalités internationales provoquent des inégalités culturelles et économiques internes entre les grands centres urbains et les populations vues comme techniquement moins avancées (autochtone, rurale). Cette structure coloniale mène à l'inégalité de développement (technique, institutionnelle et culturelle) entre les groupes « construits » vus comme évolués et leurs opposants vus comme non-évolués. 


\section{Redonner une place à l'intelligence pratique}

La structure académique universitaire doit débanaliser I'intelligence pratique dans le débat sur le développement de la conscience critique et politique. Dans son livre "L'acte est une aventure », Mendel[19] explique comment la naissance philosophique centrée sur une réflexion de l'être comme abstraction, il y a 25 siècles, a expulsé l'acte, en le remplaçant par la " pensée » sur l'acte. "Notre culture [occidentale] s'est construite à partir d'une vision triomphaliste de l'esprit humain et sur la base fallacieuse d'une capacité de domination totale de la nature et, avec les philosophies de l'histoire, de maîtrise de la société." [19 p14] Le discours sur l'action possède une fonction idéologique : il détourne le regard " de ce qui est en train de se passer » à "ce que l'on voudrait qu'il se passe ».

Le praticien pense en situation d'acte [19]. Il doit être dans I'action pour réfléchir mieux sur une situation sociale. Les rencontres avec la réalité - les risques physiques - servent de levier pour raisonner sur l'acte à entreprendre. C'est à ce moment que les organes sensoriels en tant que capteurs d'information, les savoir-faire sensorimoteurs, la perception active deviennent opératoires et non plus le raisonnement intellectuel.[19] Elle se distingue de la pensée scientifique qui s'appuie sur l'observation et la formulation de généralités basée sur des données probantes. Plus proche de la sensorialité et du corps, I'intelligence pratique est non verbale, non conceptuelle, non complètement consciente des processus de pensée qu'elle développe. Opposée à l'épistémè, la pensée pratique ne procède pas par raisonnement logique et formel; elle s'apparente à la pensée amphibie « tout-terrain » qui caractérise la « métis ».[20]

Le travail du " chercheur métis » est homologue au navigateur face à la mer. Son intervention prend la forme métis[20] aux prises avec les situations imprévisibles. II capte les opportunités d'obtenir des informations, qu'il analyse à tout moment pour rediriger ses actions. Le chercheur métis fait appel à l'intelligence navigatrice sans jamais dévier de la route qu'il a décidé de suivre. Bien qu'il sache mener droit le projet, son regard est orienté vers les points dangereux, les temps critiques et les crises inattendues où tous les renversements sont possibles. D'un côté, I'enseignement conventionnel de la science valorise la rigueur et la prévision pour assurer le succès de réalités idéalisées. De l'autre, I'enseignement alternatif discutera ouvertement de l'espace polymorphe et mouvant dans lequel le chercheur-praticien métis doit se frayer un chemin.

\section{Références}

1.Vasconcelos EM. A medicina e o pobre. [La médecine et le pauvre] São Paulo: Paulinas, 1987.

2.Vasconcelos EM. Educação popular nos serviços de saúde. [Éducation populaire dans les services de santé]. São Paulo: Hucitec, 1997.

3.Vasconcelos EM. Educação popular e a atenção à saúde da família. [Éducation populaire et attention à la santé de la famille]. São Paulo: Hucitec, 2006.

4.Laperrière $\mathrm{H}$. O olhar de uma estrangeira sobre o projeto de extensao popular. Suas analyses e percepções. [The look of a foreigner on the popular extension project]. In: Vasconcelos EM, Cruz PJSC (éds). Educação Popular na formação universitária: reflexões a partir de uma experiência [Popular education in academic training: reflections from an experience]. Sao Paulo, Brazil: Hucitec, 2011; 191-203.

5.Laperrière $H$. Expérimenter l'éducation de la libération dans le Nordeste brésilien: doutes et convictions de la recherche qualitative engagée. Recherches qualitatives 2013; Hors-Série: L'engagement du chercheur qualitatif : du porteparole au militant.

6.Valla VV. A vida religiosa como estratégia das classes populares na América Latina de superação da situação do impasse que marca suas vidas. [La vie religieuse comme stratégie des classes populaires en Amérique Latine pour dépasser la situation d'impasse marquant leurs vies.] In: Vasconcelos E (Ed.). A espiritualidade no trabalho em saúde. [La spiritualité dans le travail en santé]. São Paulo: Hucitec, 2006: 265-295.

7.Laperrière $H$, Potvin L, Zúñiga $R$. Les planifications centralisées et l'action communautaire locale. In : Aubry F, Potvin L (Éds.). L'espace socio-sanitaire. Expériences et pratiques de recherche dans la production locale de la santé. Montréal: PUM, 2012 : 227-245.

8. Nowotny H, Scott P, Gibbons M. Re-thinking science: knowledge and the public in an age of uncertainty. Cambridge: Polity, 2001.

9.Campbell DT. The experimenting society. In: Overman ES (ed). Methodology and epistemology for social science. Chicago: The University of Chicago Press, 1988: 290-314. (Originally published in 1971).

10.Bourdon Busset J, Massé P. De la prospective: Textes fondamentaux de la prospective française (1955-1966). Paris: L'Harmattan, 2007. 
11.Callon M, Lascoumes $P$, Barthe $Y$. Agir dans un monde incertain. Essai sur la démocratie technique. Paris : du Seuil, 2001.

12.Touraine A, Khosrokhavar F. La recherche de soi. Paris: Fayard, 2000.

13.Taylor C. Les sources du moi. La formation de l'identité moderne. Québec: du Boréal, 1988.

14.Gibbons $M$, Limoges $C$, Nowotny $H$ et al. The new production of knowledge. The dynamics of sciences and research in contemporary societies. London : Sage, 1994.

15.Denis JL, Lehoux P, Champagne F. Knowledge utilization in health care : from fine-tuning dissemination to contextualizing knowledge. In : Lemieux-Charles L, Champagne F. (Eds). Multidisciplinary perspectives on evidence-based decision-making in health care. Toronto : University of Toronto Press, 2004 : 11-41.

16. Lovisolo $\mathrm{H}$. Al servicio de nosotros mismos : relaciones entre agentes y campesinos. [Au services de nous-mêmes : relations entre agents et agriculteurs]. In : Tapia G. (ed). La producción de conocimientos en el medio campesino. [La production des connaissances dans le milieu agricolte]. Santiago, Chile: Programa interdisciplinario de investigaciones en Educación, 1987.

17.Casanova PG. Exploração, colonialismo e luta pela democracia na América Latina. [Exploitation, colonialisme et lutte pour la démocratie en Amérique Latine]. Petrópolis: Vozes, 2002. (Originally published in 1965].

18.Stavenhagen R. Cómo descolonizar las ciencias sociales. [Comment décoloniser les ciences sociales] [Originally published in 1971]. In: Salazar MC (ed). La investigaciónacción participativa [La recherche-action participative]. Buenos Aires, Argentina: Humanitas, 1992: 37-64.

19.Mendel G. L'acte est une aventure. Du sujet métaphysique au sujet de I'actepouvoir. Paris : La Découverte, 1998.

20.Detienne M, Vernant JP. Les ruses de I'intelligence. La métis des Grecs. Paris : Flammarion, 1975.
Pour contacter l'auteure:

Hélène Laperrière

Professeure agrégée

Université d'Ottawa

Faculté des sciences de la santé

École des sciences infirmières

451 Smyth Road

Ottawa, Ontario, $\mathrm{K} 1 \mathrm{H} 8 \mathrm{M} 5$

Canada

courriel: helene.laperriere@uottawa.ca 